\title{
Infinite regress in decision theory, philosophy of science, and formal epistemology
}

\author{
Jeanne Peijnenburg • Sylvia Wenmackers
}

Received: 31 December 2013 / Accepted: 31 December 2013 / Published online: 17 January 2014

(C) Springer Science+Business Media Dordrecht 2014

Infinite regresses have had a mixed reception in philosophy. On the one hand, they are often quickly brushed aside as being unreasonable and even preposterous. If a position or a theory is shown to engender an infinite regress, that pretty well means the end of it: an argumentum ad infinitum is as good as a reductio ad absurdum. On the other hand, the alleged absurdity of infinite regresses has been a source of inspiration and the ground for far-reaching conclusions. It led to the requirement that a regress should come to a stop and thence to the verdict that there exist such things as a Prime Mover, a First Cause, a Highest Good, or a Causa Sui.

Infinite regresses have been discussed in practically all branches of philosophy, not only in metaphysics and epistemology, but also in ethics, philosophy of mind, logic, and argumentation theory. In recent years, they have spurred a lively debate in traditional epistemology, where 'infinitism' (the idea that an infinite chain of epistemic justification is not prima facie absurd) is on its way to becoming a mature alternative to the traditional positions, foundationalism and coherentism.

The papers in the present volume are about infinite regress in decision theory, philosophy of science, and formal epistemology. We are fortunate to have brought together a number of renowned philosophers and promising young scholars:

John Barker is associate professor of Philosophy at the University of Illinois at Springfield, and chair of the Philosophy Department. His Ph.D. dissertation, defended in 1998 at Princeton University, is entitled 'The Inconsistency Theory of Truth'. His areas of interest are philosophical logic and philosophy of language (especially paradoxes and inconsistencies) with side-interests in philosophy of mind and philosophy of science.

\footnotetext{
J. Peijnenburg $(\varangle) \cdot$ S. Wenmackers

Faculty of Philosophy, University of Groningen, Oude Boteringestraat 52, 9712 GL Groningen,

The Netherlands

e-mail: jeanne.peijnenburg@rug.nl
} 
Paul Bartha is professor of Philosophy at the University of British Columbia. He received his B.Sc. in Mathematics from the University of Toronto and his Ph.D. from the Philosophy Department at the University of Pittsburgh. He works mainly in philosophy of science and decision theory, with particular attention to issues relating to probability and confirmation. His current research concerns analogical reasoning, the role of symmetry in probabilistic reasoning, and Pascal's Wager.

Benjamin Bewersdorf is a postdoctoral researcher at the Faculty of Philosophy of the University of Groningen in the Netherlands, affiliated to a project on Infinite Regress, funded by the Netherlands Organization for Scientific Research (NWO). In 2012, he received his Ph.D. at the University of Konstanz in Germany with a thesis on belief revision. His main research interests are formal epistemology and general philosophy of science.

Alan Hájek is professor and Head of the School of Philosophy at the Research School of Social Sciences at the Australian National University. He studied mathematics and statistics at the University of Melbourne, and philosophy at the University of Western Ontario and at Princeton University. His many publications are mainly in the areas of foundations of probability theory, decision theory, formal epistemology, philosophical logic, and philosophical methodology.

Frederik Herzberg is assistant professor at the Center for Mathematical Economics at Bielefeld University, where he obtained his Habilitation in economic theory. He took a Ph.D. in nonstandard probability theory at Bonn University, and one on option pricing at Oxford University. His recent publications are about coherence, judgement aggregation, and continuous-time stochastic processes based on infinitesimals.

Hanti Lin is a postdoctoral fellow in the School of Philosophy at the Australian National University. In 2013, he received his Ph.D. from Carnegie Mellon University with a thesis on propositional reasoning that tracks probabilistic reasoning. He works in formal epistemology and on the foundations of decision theory, with applications to philosophy of science (especially Ockham's razor) and philosophy of language (including expressivism in metaethics).

John D. Norton is professor in the Department of History and Philosophy of Science at the University of Pittsburgh. Since 2005, he is also the director of the Center for Philosophy of Science, a meeting place for philosophers of science from all over the world. John Norton has published extensively on the history and the philosophy of science, with an emphasis on relativity theory, both special and general, statistical physics, thought experiments, causation, induction and confirmation.

We thank the authors for contributing to this issue and all referees for providing us with valuable comments and timely reports.

Editorial work for the present volume has been carried out with funding from the Netherlands Organization for Scientific Research (NWO), grant number 360-20-280 (Jeanne Peijnenburg's Free Competition subsidy for research on infinite regresses in epistemology) and grant number 639.031.244 (Sylvia Wenmackers' VENI subsidy 'Inexactness in the Exact Sciences' for research on infinitesimals). We are grateful for this support. 